\title{
Arthroscopic Knotless Separate Layer Transosseous Equivalent Repair of Delaminated Rotator Cuff Tears
}

\author{
Kadir Buyukdogan, M.D., Ozgur Koyuncu, M.D., Ilker Eren, M.D., Olgar Birsel, M.D., \\ Michael A. Fox, B.A., and Mehmet Demirhan, M.D.
}

\begin{abstract}
Delamination of rotator cuff tears presents a challenge for surgeons. Recognizing and repairing such a complex tear pattern often require innovative approaches to achieve an anatomic restoration of footprint. In this Technical Note, we described our preferred method that anatomically repairs both layers of delaminated rotator cuff tear separately in a knotless transosseous equivalent technique. Two sutures are placed to the articular layer in a cinch stitch configuration. Then, closed-loop end sutures are passed through both layers while keeping the closed-loop end at the working portal. The free ends of cinch stitches are loaded to anchors with a preloaded fiber tape loop, which is placed to the medial row while approximating the articular layer onto its footprint. Fiber tapes are then shuttled through both layers of tendon with the help of a previously placed closed-loop suture. Finally, the lateral row anchors are placed while fiber tapes are tensioned in a cross-bridge configuration. We believe that this technique may facilitate uneventful healing of delaminated rotator cuffs by providing the biomechanical properties of transosseous equivalent repair.
\end{abstract}

\section{Introduction}

$\mathbf{T}$ he outcome of rotator cuff repairs is influenced by several factors, including bone quality, repair technique, tear pattern, and biological milieu of the torn tendon. Delaminated tears of the rotator cuff have been considered a form of degeneration within the tendon and described as a horizontal split of the tendon substance with vascular changes. ${ }^{1}$ Delamination of the rotator cuff tears is gaining attention in the literature and probably occurs more frequently than appreciated. In previous studies, the prevalence of delamination varied from $33 \%$ to $92 \%$, depending on the surgical

From the Department of Orthopedic Surgery, Koc University School of Medicine (K.B., I.E., O.B., M.D.), Zeytinburnu/Istanbul, Turkey; Department of Orthopaedic Surgery, VKV American Hospital (O.K.), Teşvikiye, Sisli/ Istanbul, Turkey; and University of Virginia School of Medicine (M.A.F.), Charlottesville, Virginia, U.S.A.

The authors report that they have no conflicts of interest in the authorship and publication of this article and that no funding or financial remuneration was received. Full ICMJE author disclosure forms are available for this article online, as supplementary material.

Received April 24, 2019; accepted June 16, 2019

Address correspondence to Kadir Buyukdogan, M.D., Department of Orthopaedic Surgery, Koc University Hospital, Davutpasa Street, No. 4, Zeytinburnu/Istanbul 34010,Turkey.E-mail:kbuyukdogan@gmail.com

(C) 2019 by the Arthroscopy Association of North America. Published by Elsevier. This is an open access article under the CC BY-NC-ND license (http:// creativecommons.org/licenses/by-nc-nd/4.0/).

2212-6287/19547

https://doi.org/10.1016/j.eats.2019.06.008 methods, number of cases, and preference of viewing portals. $^{2-6}$

The rotator cuff is composed of 5 layers defined by the attachments and orientations of the fibrous elements in each of these layers. Two major layers of the superficial bursal and deeper articular sides are clinically differentiated. ${ }^{7}$ When delamination occurs, the deeper layer is typically more retracted than the superficial layer. ${ }^{6}$ Although the cause of delamination is still unclear, local ischemia and uneven stress distribution between the layers of cuff have been postulated as causes of delamination. ${ }^{1}$ Further, studies have shown delaminated tears of the rotator cuff are lined by a layer of synovial-like cells, which facilitates movement between the layers of delamination and prevents the layers from healing together. ${ }^{1}$ There is an ongoing debate regarding whether the articular layer is a real articular layer of the supraspinatus or infraspinatus, a superior capsule complementing the rotator cuff insertion, or a rotator cable. ${ }^{2,3,8,9}$ Regardless of its anatomic definition, the restoration of both the articular and bursal layers onto the footprint with consideration of the retraction pattern and repair tension of each layer is believed to be of most importance to preserve structural integrity after cuff repair. ${ }^{10-14}$

Delaminated rotator cuff tears pose technical difficulties for anatomic repair and probably have less favorable outcomes. Several reports have suggested that the presence of delaminated tears was a negative 

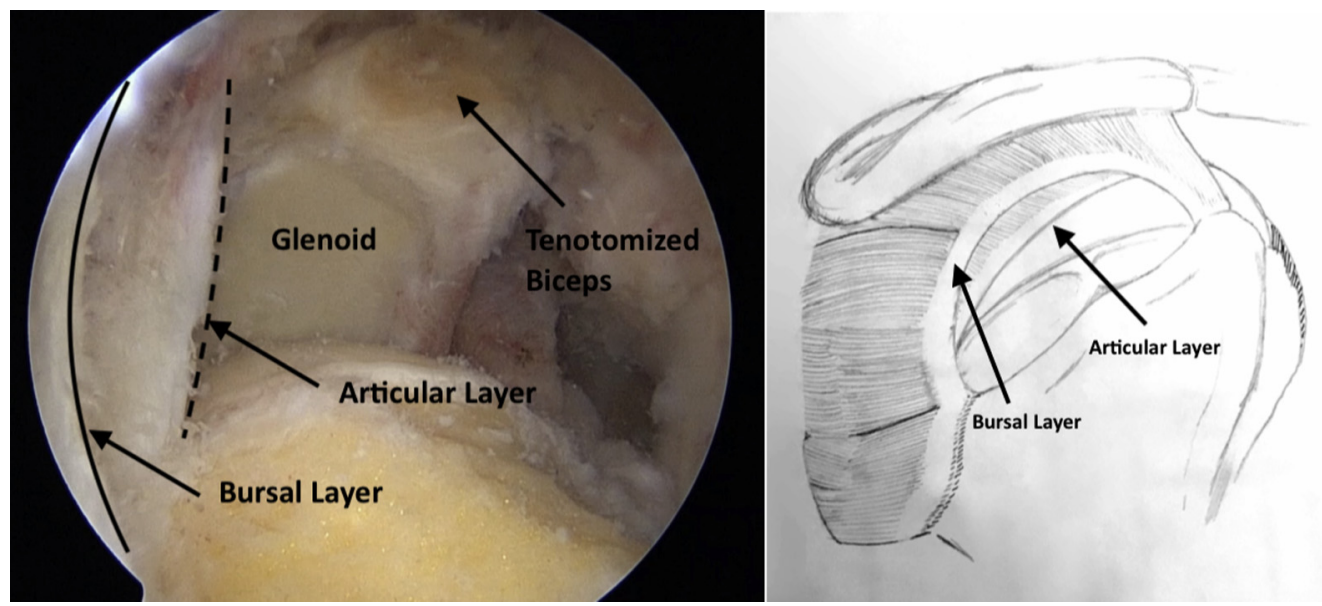

Fig 1. Arthroscopic view of the right shoulder through the lateral portal in beach-chair position. All adhesions on the articular and bursal layers of the rotator cuff are released down to the coracoid base to improve tendon mobility. Tear morphology and extent of delamination are confirmed.

prognostic factor in functional and radiologic results after rotator cuff repair. ${ }^{4,5,12}$ To improve outcomes after the repair of delaminated rotator cuff tears, numerous techniques of suturing each laminated layer separately have been introduced. ${ }^{11,15-17}$ The purpose of this report is to describe our preferred technique of anatomic repair of both layers of delaminated rotator cuff tears separately in a knotless transosseous equivalent technique while securing the articular layer with cinch sutures to provide additional stability.

\section{Surgical Technique}

Surgery is performed with the patient under general anesthesia with an additional interscalene nerve block. An examination with the patient under anesthesia is carried out before arthroscopy to evaluate stiffness. The patient is placed in beach-chair position with a pneumatic arm holder (Trimano Fortis; Arthrex, Naples, FL), and the index shoulder is prepared and draped in a sterile fashion. A standard posterior viewing portal is established, and immediately an anterior working portal with a cannula (Crystal Cannula; Arthrex) is placed in the rotator interval. A comprehensive diagnostic arthroscopy is carried out to address any underlying intra-articular pathologies, including anterior and posterior capsule adhesions, subscapularis tendon tears, and biceps tendon pathologies. If needed, subscapularis tendon repair and biceps tenotomy or tenodesis are performed in this step.

Next, the anterolateral portal is placed just anterior to the anterolateral corner of the acromion with use of a spinal needle. An arthroscopic shaver (APS II Handpiece; Arthrex) is inserted through the anterolateral portal, and the rotator cuff tear is inspected and debrided while the arthroscope remains in the glenohumeral joint via the posterior portal. Any spurs on the greater tuberosity are gently removed to achieve a smooth surface, with the use of an open curette and shaver
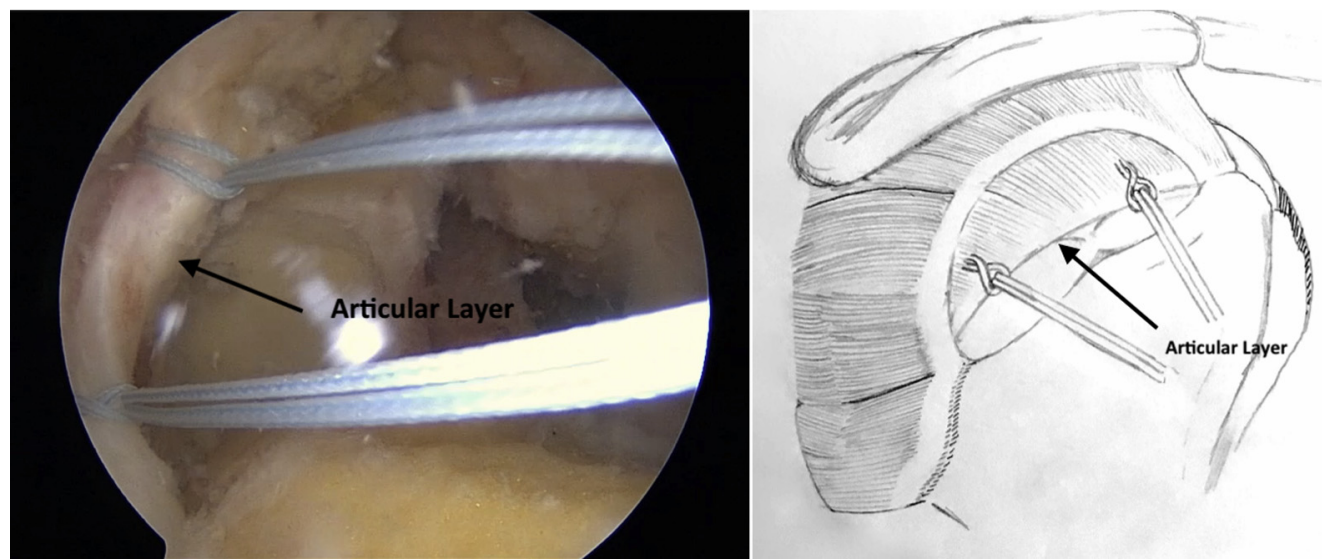

Fig 2. Right shoulder viewed from lateral portal. With the help of a self-retrieving suture passer, a No. 2 FiberWire is passed through the articular layer and retrieved out the anterolateral portal as a loop. Two free ends of the FiberWire are threaded through the loop to create a cinch configuration. Free ends are pulled through the anterolateral portal, and the cinch is slid down on the articular layer. This process is repeated, so there are 2 No. 2 FiberWire cinch stitches in place in the articular layer. 

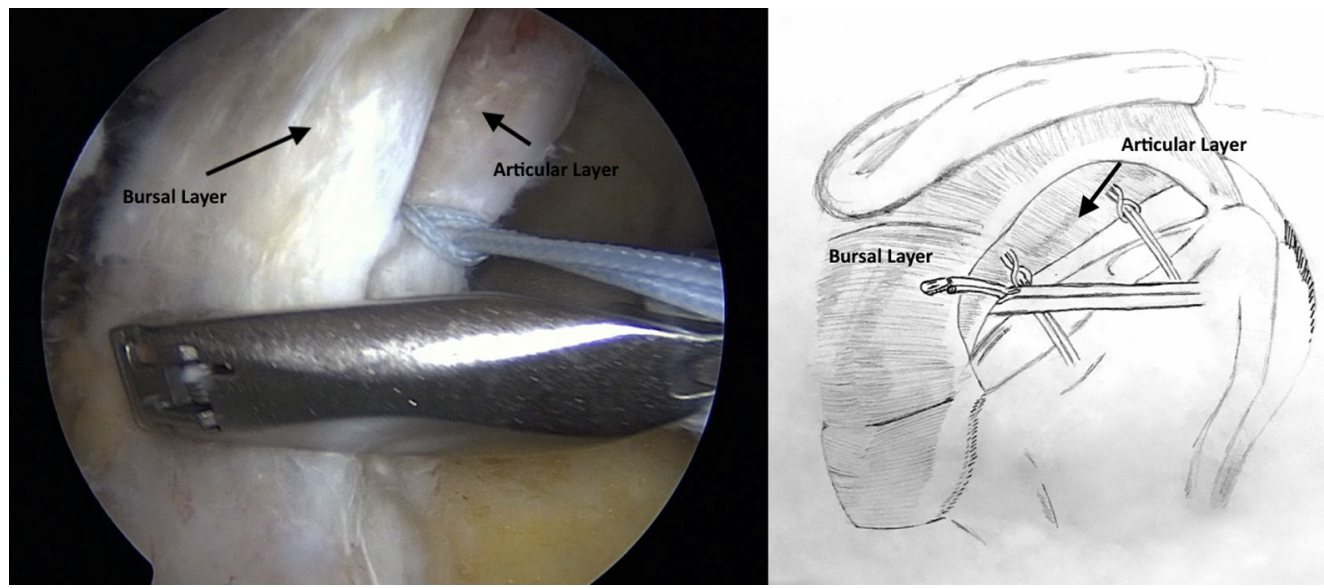

Fig 3. View from right shoulder lateral portal with a self-retrieving suture passer in the anterolateral portal. Suture passer is already loaded with a FiberLink suture and bites articular and labral layers simultaneously.

(Bone Cutter; Arthrex). Then, the arthroscope is removed from the glenohumeral joint and placed into the subacromial space through the same posterior skin incision. A lateral portal is established 1 fingerbreadth distal to the anterolateral acromion with use of a spinal needle, and a large threaded cannula (Twist-In Cannula; Arthrex) is introduced under direct visualization (outside-in technique). A thorough subacromial decompression is performed, and the coracoacromial ligament is released from the acromion with the use of an electrocautery device (Arthrocare; Smith \& Nephew, Memphis, TN). Acromioplasty is performed by using a burr to achieve a flat acromion for type 2 and type 3 acromia. The acromioclavicular ligament is visualized, and coplaning of distal clavicula is performed if any inferior spurs are encountered on the distal clavicle. Distal clavicle resection may be performed to address acromioclavicular joint pathologies.

The arthroscope is then introduced into the lateral portal, and a second large-diameter threaded cannula (Twist-In Cannula; Arthrex) is placed into the posterior portal. The anterolateral portal is moved to the subacromial space, and a PassPort cannula (Arthrex) is placed. Then, the footprint of the rotator cuff is identified and cleared of soft tissue while avoiding excessive decortication, during viewing through the lateral portal.

Once all of the adhesions are removed and rotator cuff release is completed, tear morphology and extent of the delamination are confirmed through the lateral viewing portal (Video 1, Fig 1). Retractability and tension of each layer are evaluated for compliance with the separate layer repair technique. If the layers are fragile or overretracted, additional release techniques should be used or standard double-row fixation should be performed. After confirmation of the quality of the layers, a probe can be placed into the delamination site to determine the size of the defect. A shaver can be inserted into the defect to abrade the opposing surfaces and stimulate healing. ${ }^{1,6}$

A No. 2 FiberWire (Arthrex) is folded in the midportion and loaded into a self-retrieving suture passer (FastPass Scorpion SL; Arthrex). While viewing from lateral portal,
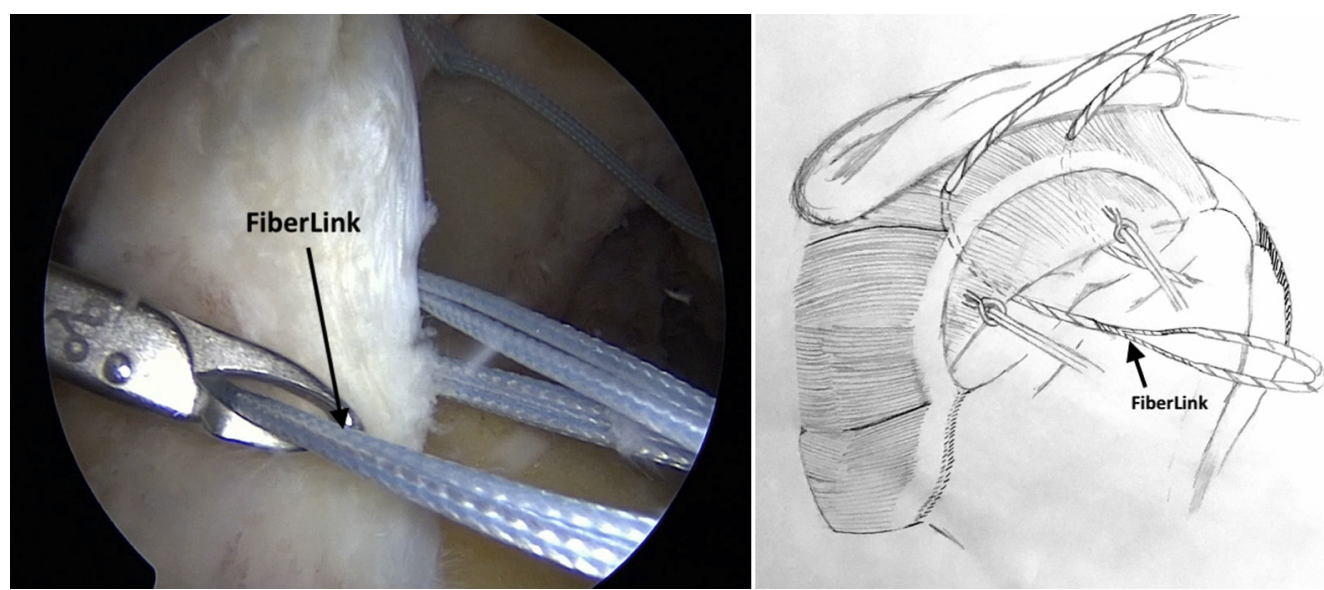

Fig 4. View from right shoulder lateral portal. Single end of FiberLink suture is retrieved through posterior portal, while keeping the closed-loop end at the anterolateral portal for shuttling fiber tapes in the following steps. 

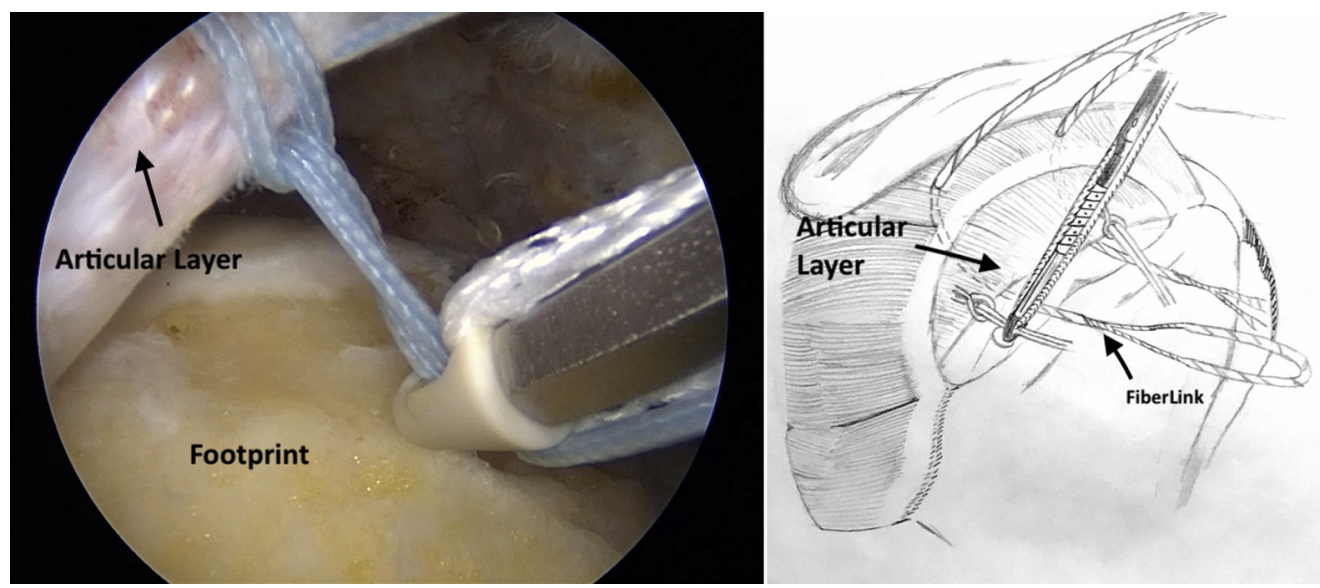

Fig 5. View from right shoulder lateral portal demonstrating free ends of the cinch stitch loaded into eyelet of 4.75 -mm biocomposite SwiveLock anchor with FiberTape Loop. Tension is applied to free ends of cinch through eyelet.

a No. 2 FiberWire is passed from the posterior portion of the articular layer and retrieved from the anterolateral portal. The suture passer releases the FiberWire as a loop outside the portal. Then, 2 free ends of the No. 2 FiberWire suture are threaded through this loop to create a cinch configuration as the free ends are pulled to slide the cinch down to the articular layer. These steps are repeated for the anterior portion so that 2 cinch stiches are placed in the articular layer (Fig 2). Instead of No. 2 FiberWire sutures, FiberLink sutures (Arthrex) with a closed loop on 1 end may also be used to create cinch stiches in this step.

Then, the rotator cuff tendons that have been pulled with a grasper and a FiberLink suture are passed through the posterior portion of both articular and bursal layers simultaneously just lateral to the musculotendinous junction (Fig 3). The single end is retrieved through the posterior portal, while keeping the closedloop end at the anterolateral portal to shuttle fiber tapes (Fig 4). Thereafter, the insertion spots for medial anchors are determined while keeping at least a 15- to 20- $\mathrm{mm}$ bone bridge between anchors. The posterior socket for the medial row is placed in the medial portion of the greater tuberosity just beneath the articular cartilage and prepared with an arthroscopic screw tap.

The free ends of the posterior cinch stitch are loaded into the eyelet of a $4.75-\mathrm{mm}$ BioComposite vented SwiveLock anchor with FiberTape Loop (Arthrex). The anchor is placed into the medial row while suture limbs of cinch stitch are tensioned through the eyelet of the SwiveLock anchors (Fig 5). Now, the posterior portion of the articular layer is compressed down onto its insertion site at the bone-cartilage margin, and the free ends of the cinch stitch are cut. FiberTape Loop is threaded through the closed loop of FiberLink, which was previously passed through the articular and bursal layers of the cuff (Fig 6). Then, FiberLink suture is used to shuttle FiberTape through both layers of the rotator cuff (Fig 7). These steps are repeated for the anterior portion of the tear to reduce the articular layer onto its footprint (Fig 8).
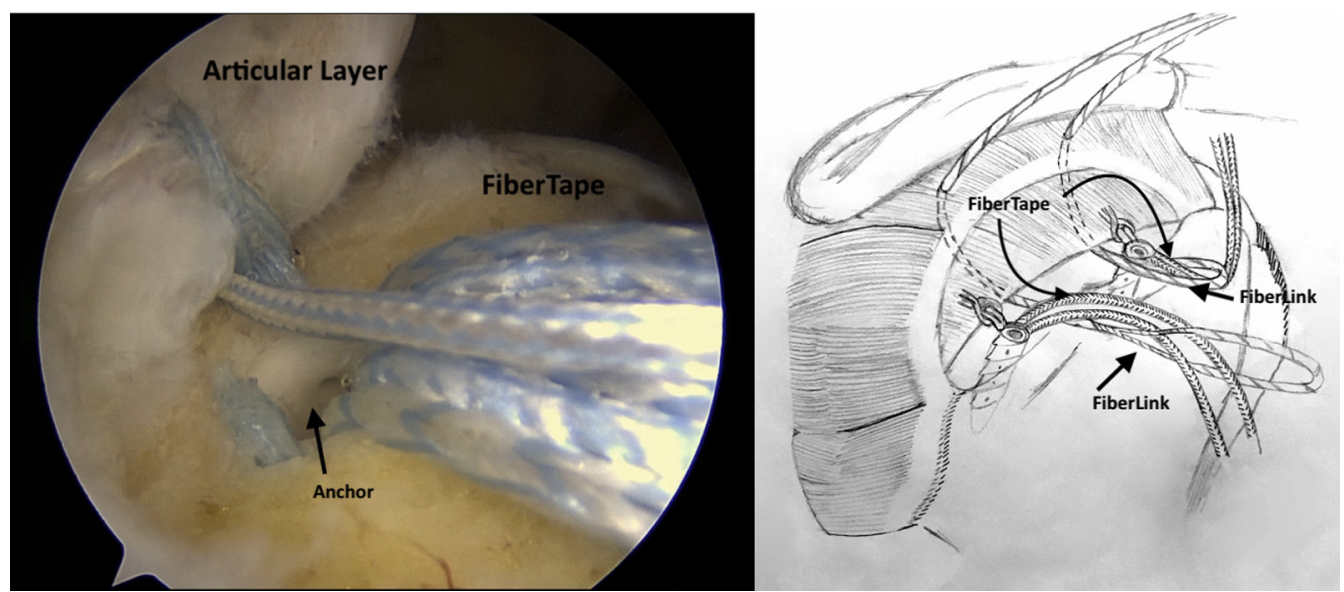

Fig 6. Right shoulder viewed from lateral portal. Extracorporeally, FiberTape sutures are threaded through the closed loop of the FiberLink, which was previously passed through both layers of the rotator cuff. Then, FiberLink sutures are used to shuttle fiber tapes through both layers of rotator cuff. 

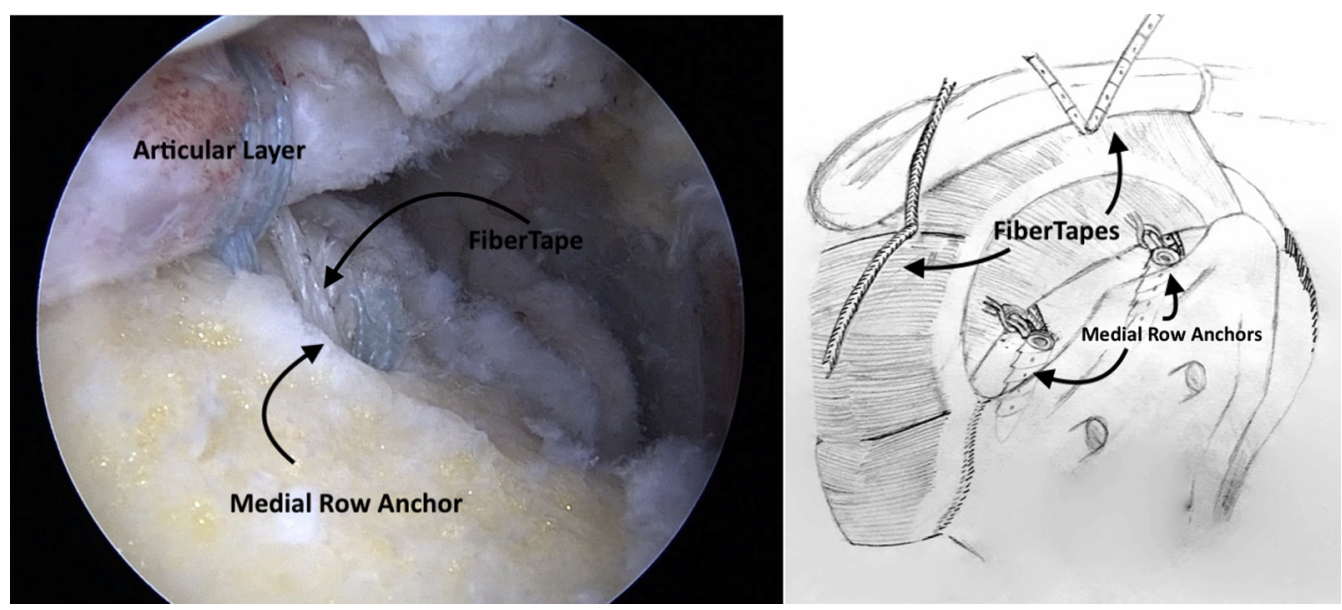

Fig 7. Arthroscopic view of the right shoulder through lateral portal shows the medial row after passing the suture tapes underneath the articular layer and retrieving them from the top of the bursal layer.

The spliced tails of the tapes are cut off, and 1 fiber tape from each medial row is loaded into $5.5-\mathrm{mm}$ biocomposite knotless anchors (SwiveLock; Arthrex). Lateral row sockets are placed distal to the lateral edge of the footprint with the use of a bone punch. Lateral row anchors are placed while fiber tapes are tensioned adequately in a cross-bridge configuration. If dog-ear deformity is anticipated, an additional FiberLink or No. 2 FiberWire tip retention suture of SwiveLock anchors can be passed through the deformity in a cinch configuration and loaded into the anchor with FiberTape before fixation of the lateral row (Fig 9). Pearls and pitfalls of the described technique are outlined in Table 1.

\section{Rehabilitation}

A rotator cuff repair protocol is used postoperatively, and the patient is placed in an arm sling with an abduction pillow. Tabletop activities without the sling are allowed immediately after surgery. Core and periscapular exercises are also initiated immediately. The arm sling is maintained for 4 weeks after surgery.
Thereafter, active assistive range of motion and stick exercises begin 4 to 5 weeks postoperatively. Strengthening and active resistance exercises are encouraged after 3 months. Full return to activities, including sports and heavy labor, is allowed after 6 months.

\section{Discussion}

Previous studies reported that the presence of delamination at time of repair is a negative prognostic factor for the outcome of arthroscopic rotator cuff repair. ${ }^{4,5}$ Considering that delamination can affect structural integrity after cuff repair, the anatomic restoration of both articular and bursal layers onto the footprint is important. When delamination occurs, the articular layer is typically more retracted than the bursal layer, and this retraction pattern may necessitate complex repair techniques. ${ }^{1,14-17}$ In this Technical Note, we described and discussed our preferred knotless separate layer transosseousequivalent technique to repair delaminated rotator cuff tears.

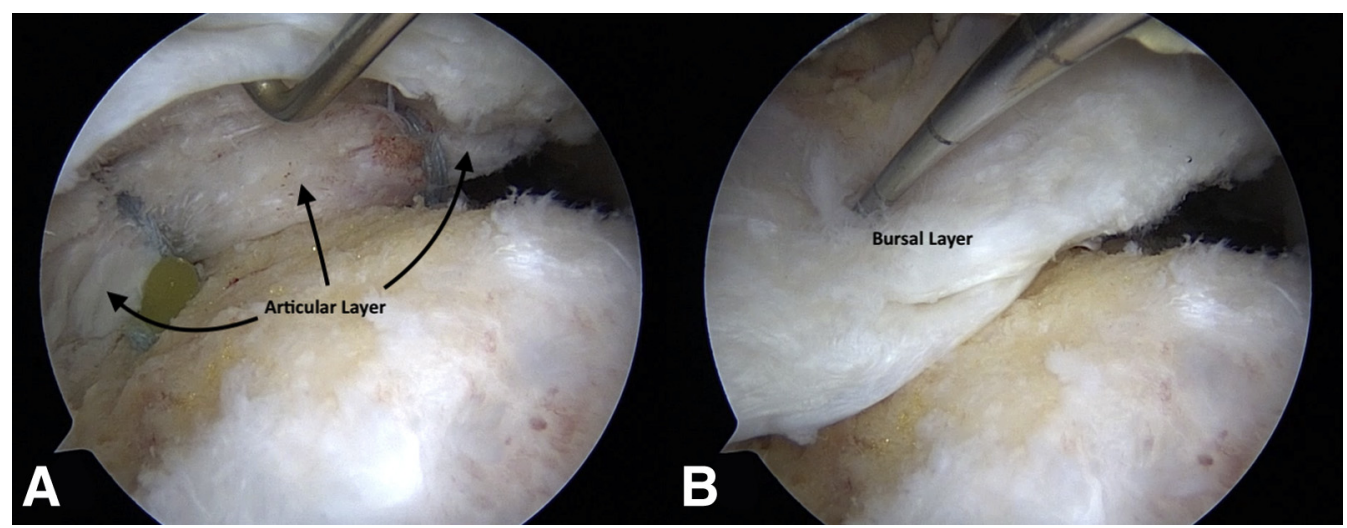

Fig 8. (A) Arthroscopic view of the right shoulder rotator cuff footprint from the lateral portal depicts the articular layer is secured onto its footprint. (B) Bursal layer shows adequate excursion to cover footprint completely. 


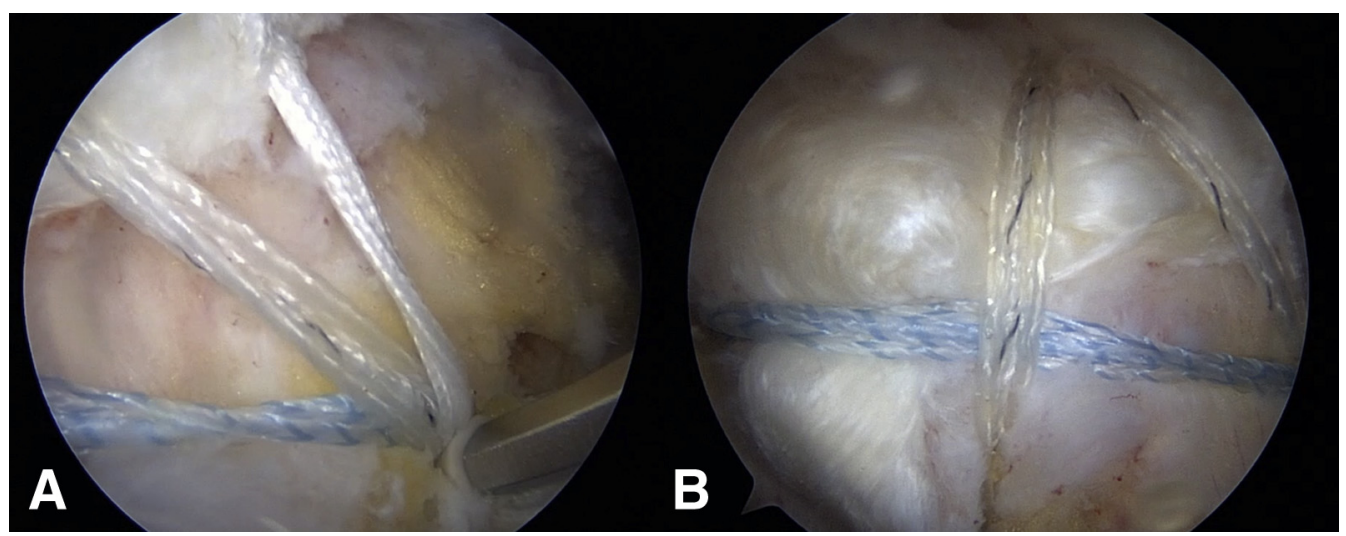

Fig 9. (A) Right shoulder viewed from lateral portal. If dog-ear deformity is anticipated, an additional FiberLink can be passed through the deformity in a cinch stich configuration and loaded into the anchor with fiber tapes before fixation of lateral row. (B) FiberTape sutures are then secured laterally with a knotless suture anchor in a transosseous equivalent manner.

Layered structure of the rotator cuff has been studied extensively. ${ }^{7}$ However, the exact location where the delamination occurs is still a matter of debate. In an immunochemical study by Sonnabend et al., ${ }^{1}$ the authors proposed that delamination occurs between zone 2 and zone 3 of the rotator cuff. Han et al. ${ }^{3}$ reported that the retracted articular layer is the rotator cable, which is separated from the cuff. In an anatomic study, Nimura et al. ${ }^{8}$ stated that the articular capsule occupies a substantial area of the greater tuberosity and suggested that the articular layer of the delaminated lesion mainly involves the articular capsule. Recently, Adams et al. ${ }^{9}$ reported that rotator cuff repair must restore the normal capsular anatomy to provide normal

Table 1. Surgical Pearls and Pitfalls

\section{Pearls}

Viewing through the lateral portal may allow for recognition of cuff tear pattern.

While preparing the footprint, avoid excessive abrasion of bone to minimize possibility of suture anchor pull-out.

Using self-retrieving suture passers may allow precise passage of sutures through the tendon.

Placing cannulas to working portals facilitates suture passing.

Working from outside in helps avoid the formation of dog-ears.

To avoid the potential for dog-ear formation, additional cinch

stitches may be passed through the deformity and loaded into the lateral row anchor.

Pitfalls

Improper visualization and failure to recognize tear pattern may lead to nonanatomic repair.

If the layers are fragile and immobile, this procedure should be avoided.

Aggressive release of the articular layer from the glenoid attachment may lead to capsular damage.

There is possible suprascapular nerve injury if the debridement and release are carried too medial to glenoid rim.

When passing sutures through rotator cuff, stay lateral to the musculotendinous junction.

Avoid excessive traction when shuttling FiberTape sutures under the articular layer. biomechanics of the joint. which ultimately is responsible for good clinical outcomes.

The directions in which the deep and superficial layers retract may differ in delaminated cuffs, and there may be tension mismatch during the repair of a delaminated rotator cuff tear if the layers are tied and repaired together. ${ }^{1,12}$ Therefore, several authors recommended that 2 layers be repaired in different manners to withstand the nonhomogeneous strains occurring during shoulder movement. ${ }^{10-12,15-17}$ Briefly, the articular layer containing the articular capsule should be attached to the footprint of the capsule at the medial edge of the greater tuberosity. In contrast, the superficial layer should be shifted laterally to restore the appropriate force couple balance required in the glenohumeral fulcrum. ${ }^{17}$

Regarding the clinical outcome of a rotator cuff tear with delamination, Sugaya et al. ${ }^{10}$ reported that separate layer repair using double-row fixation improved cuff integrity. Recently, Opsomer et al. ${ }^{11}$ published their results after arthroscopic double-layer lasso loop repair technique for delaminated posterosuperior rotator cuff tears. Their series showed a low rerupture rate and good to excellent recovery with resultant functional, pain, and patient satisfaction scores. Kim et al. ${ }^{12}$ compared the clinical outcomes between en masse suture bridge repair and separate repair for the treatment of rotator cuff with delamination and reported improved clinical outcomes in both repair groups, but the separate repair group had lower pain scores. In a recent study, Nakamizo and Horie ${ }^{14}$ compared clinical outcomes between 2 suturing procedures-en masse and dual-layer suture bridging-for delaminated rotator cuff tears. Both procedures improved clinical and radiographic outcomes, but the dual-layer suture bridge technique achieved better postoperative range of motion. ${ }^{14}$

The technique described here has some pros and cons, which outlined in Table 2. Unlike the previously 
Table 2. Advantages and Disadvantages of the Arthroscopic Knotless Double-Layer Transosseous Equivalent Repair Technique

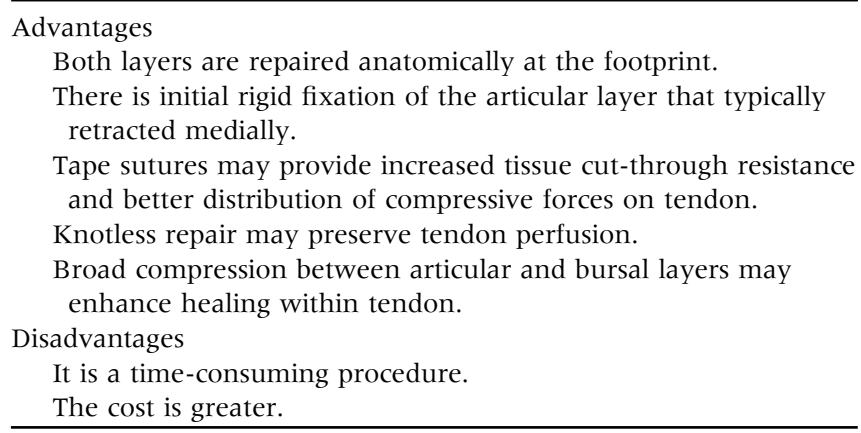

reported separate layer techniques, the most important feature of this technique is that, after repairing the articular layer with cinch stitches in a knotless fashion, it allows to repair the entire delaminated cuff in a transosseous equivalent manner with suture tapes. Opsomer et al. ${ }^{11}$ described a unique suture technique that allows locking of the deep tendon layer and securing it to the medial aspect of the footprint with lasso loops. In a Technical Note description followed by a biomechanical study, Pauzenberger et al. ${ }^{13}$ and Heuberer et al. ${ }^{15}$ introduced a novel technique that affixes the articular layer onto the footprint by the use of loop sutures (FiberLink) in cinch configuration, while approximating the bursal layer to the more lateral aspect of the footprint to restore normal footprint anatomy. In these reports, including ours, avoiding knots between the layers may have some benefits to promote increased interlayer contact between the fibers, thus enhancing the healing process. ${ }^{11,17}$ Furthermore, biomechanical studies suggest that selfcinching stitches lead to superior tissue-holding strength at the tissue-suture interface compared with equivalent non-self-cinching stitches. ${ }^{18}$

In contrast with the double-row techniques, which secure the tendon only at the anchor fixation points, transosseous equivalent repair allows compression of the tendon about the entire length of suture from medial to lateral fixation. Previous studies have emphasized the self-reinforcement and interconnectivity properties of transosseous equivalent repairs that allow a higher ultimate load to failure, increased contact area, and pressure with minimizing gap formation. ${ }^{19-21}$ Our technique offers an anatomic reduction of both layers on the footprint and may provide the previously established advantages of transosseous equivalent repair. After initial fixation of the articular layer onto the footprint under proper tension, using a transosseous equivalent repair may unite the delaminated tear as a single construct to minimize shear forces between layers. In addition to improved biomechanical characteristics of transosseous equivalent repair, avoidance of medial knot tying is proposed to prevent tendon strangulation and medial row failure, ${ }^{21-23}$ and thus may be attributed as another advantage of our technique. Furthermore, using tape sutures rather than conventional sutures may help to better distribute compressive forces on the cuff tendons and may improve tissue cut-through resistance. ${ }^{19-22}$ However, there are some potential disadvantages of this technique as seen in other separate layer repairs. Higher cost and prolonged operative time may be considered as the major drawbacks of this technique. It is a highly technical procedure with several steps, but knotless fixation of the rotator cuff may ease the repair and eliminate the time for knot tying.

We believe that our technique may restore normal biomechanics of the shoulder joint and facilitate uneventful healing of delaminated rotator cuffs by providing the aforementioned biomechanical properties of transosseous equivalent repair. However, clinical and comparative biomechanical data are still lacking to support the hypothesized advantages of our technique.

\section{Acknowledgment}

The authors thank Recep Akbulut for his valuable contribution to the technical illustrations.

\section{References}

1. Sonnabend DH, Yu Y, Howlett CR, Harper GD, Walsh WR. Laminated tears of the human rotator cuff: A histologic and immunochemical study. J Shoulder Elbow Surg 2001;10:109-115.

2. MacDougal GA, Todhunter CR. Delamination tearing of the rotator cuff: Prospective analysis of the influence of delamination tearing on the outcome of arthroscopically assisted mini open rotator cuff repair. J Shoulder Elbow Surg 2010;19:1063-1069.

3. Han Y, Shin JH, Seok CW, Lee CH, Kim SH. Is posterior delamination in arthroscopic rotator cuff repair hidden to the posterior viewing portal? Arthroscopy 2013;29:17401747.

4. Flurin P-H, Landreau P, Gregory T, et al. Cuff integrity after arthroscopic rotator cuff repair: Correlation with clinical results in 576 cases. Arthroscopy 2007;23:340-346.

5. Boileau P, Brassart N, Watkinson DJ, Carles M, Hatzidakis AM, Krishnan SG. Arthroscopic repair of fullthickness tears of the supraspinatus: Does the tendon really heal? J Bone Joint Surg Am 2005;87:1229-1240.

6. Sonnabend DH, Watson EM. Structural factors affecting the outcome of rotator cuff repair. J Shoulder Elbow Surg 2002;11:212-218.

7. Clark JM, Harryman DT 2nd. Tendons, ligaments, and capsule of the rotator cuff. Gross and microscopic anatomy. J Bone Joint Surg Am 1992;74:713-725.

8. Nimura A, Kato A, Yamaguchi K, et al. The superior capsule of the shoulder joint complements the insertion of the rotator cuff. J Shoulder Elbow Surg 2012;21:867-872. 
9. Adams CR, DeMartino AM, Rego G, Denard PJ, Burkhart SS. The rotator cuff and the superior capsule: Why we need both. Arthroscopy 2016;32:2628-2637.

10. Sugaya H, Maeda K, Matsuki K, Moriishi J. Repair integrity and functional outcome after arthroscopic double-row rotator cuff repair. A prospective outcome study. J Bone Joint Surg Am 2007;89:953-960.

11. Opsomer GJ, Gupta A, Haeni DL, et al. Arthroscopic double-layer lasso loop technique to repair delaminated rotator cuff tears. Arthroscopy 2018;34:2943-2951.

12. Kim YS, Lee HJ, Jin HK, Kim SE, Lee JW. Conventional en masse repair versus separate double-layer double-row repair for the treatment of delaminated rotator cuff tears. Am J Sports Med 2016;44:1146-1152.

13. Pauzenberger L, Heuberer PR, Dyrna F, et al. Doublelayer rotator cuff repair: Anatomic reconstruction of the superior capsule and rotator cuff improves biomechanical properties in repairs of delaminated rotator cuff tears. Am J Sports Med 2018;46:3165-3173.

14. Nakamizo H, Horie R. Comparison of en masse versus dual-layer suture bridge procedures for delaminated rotator cuff tears. Arthroscopy 2018;34:3150-3156.

15. Heuberer PR, Pauzenberger L, Smolen D, Ostermann RC, Anderl W. An arthroscopic knotless technique for anatomical restoration of the rotator cuff and superior capsule: The double-layer cinch bridge. Arthrosc Tech 2018;7:e7-el2.

16. Mochizuki T, Nimura A, Miyamoto T, Koga H, Akita K, Muneta T. Repair of rotator cuff tear with delamination: Independent repairs of the infraspinatus and articular capsule. Arthros Tech 2016;5:e1129-el134.

17. Hepp P, Engel T, Osterhoff G, Marquass B, Josten C. Knotless anatomic double-layer double-row rotator cuff repair: A novel technique re-establishing footprint and shape of full-thickness tears. Arch Orthop Trauma Surg 2009;129:1031-1036.

18. Ponce BA, Hosemann CD, Raghava P, Tate JP, Eberhardt AW, Lafosse L. Biomechanical evaluation of 3 arthroscopic self-cinching stitches for shoulder arthroscopy: The lasso-loop, lasso-mattress, and double-cinch stitches. Am J Sports Med 2011;39:188-194.

19. Park MC, Tibone JE, El Attrache NS, Ahmad CS, Jun BJ, Lee TQ. Part II: Biomechanical assessment for a footprint-restoring transosseous-equivalent rotator cuff repair technique compared with a double-row repair technique. J Shoulder Elbow Surg 2007;16:469476.

20. Park MC, El Attrache NS, Tibone JE, Ahmad CS, Jun BJ, Lee TQ. Part I: Footprint contact characteristics for a transosseous-equivalent rotator cuff repair technique compared with a double-row repair technique. J Shoulder Elbow Surg 2007;16:461-468.

21. Burkhart SS, Adams CR, Burkhart SS, Schoolfield JD. A biomechanical comparison of 2 techniques of footprint reconstruction for rotator cuff repair: The SwiveLockFiberChain construct versus standard double-row repair. Arthroscopy 2009;25:274-281.

22. Barber FA, Drew OR. A biomechanical comparison of tendon-bone interface motion and cyclic loading between single-row, triple-loaded cuff repairs and double-row, suture-tape cuff repairs using biocomposite anchors. Arthroscopy 2012;28:1 197-1205.

23. Millett PJ, Espinoza C, Horan MP, et al. Predictors of outcomes after arthroscopic transosseous equivalent rotator cuff repair in 155 cases: A propensity score weighted analysis of knotted and knotless self-reinforcing repair techniques at a minimum of 2 years. Arch Orthop Trauma Surg 2017;137:1399-1408. 